ISSN electrónico: 2602-8069

\title{
¿HACIA UN MODELO AGROALIMENTARIO ÚNICO? DIVERSIDAD E IDENTIDADES ESPACIALES EN EL CONSUMO DE ALIMENTOS EN ECUADOR
}

Towards an unified agro-food model?

Spatial diversity analysis of the food consumption in Ecuador

Fernando Barragán-Ochoa

Investigador asociado al laboratorio PRODIG

Miembro de la Asociación Geográfica del Ecuador

fbarraganochoa@gmail.com

Fecha de recepción del artículo: 30/03/2019

Fecha de aceptación definitiva: 13/06/2019 


\section{¿HACIA UN MODELO AGROALIMENTARIO ÚNICO? DIVERSIDAD E IDENTIDADES ESPACIALES}

EN EL CONSUMO DE ALIMENTOS EN ECUADOR

\section{RESUMEN}

El presente artículo examina la espacialización del consumo de alimentos en Ecuador para determinar en qué medida el desarrollo del modelo agroindustrial a nivel global ha generado un modelo único de alimentación. En efecto, las tendencias alimentarias globales habrían borrado las diferencias espaciales en el consumo de alimentos, un tema crucial que se contrapone a los fundamentos de la geografía de la alimentación. En una primera sección, se estudia el régimen alimentario ecuatoriano prestando atención a su evolución temporal y a las similitudes y diferencias frente a sus vecinos (Colombia y Perú), América del Sur y el mundo. En la segunda sección, para comprender los diferentes regímenes alimentarios al interior del país, se evalúa la presencia o ausencia de alimentos en las alacenas de los hogares ecuatorianos por provincia con datos provenientes de la Encuesta Nacional de Ingresos y Gastos de Hogares Urbanos y Rurales (ENIGHUR). Finalmente, en la tercera sección, la interacción del comportamiento espacial de los 27 productos de mayor presencia y variación espacial permite generar cinco regiones alimentarias caracterizadas por un consumo similar. El estudio permite concluir que si bien el modelo alimentario agroindustrial forma parte de las tendencias que han modificado los hábitos de consumo de los ecuatorianos, hay una expresión espacial de los regímenes alimentarios a manera de regiones. Estas regiones responden a factores biofísicos, pero sobre todo a procesos sociales de la construcción del espacio nacional, que tienen también una impronta en los elementos culturales.

Palabras clave: modelo agroalimentario, geografía de la alimentación, relaciones campo ciudad, Ecuador, demanda alimentaria, abastecimiento alimentario

\section{ABSTRACT}

This paper examines the spatialization of food consumption in Ecuador in order to determine if the development of the global agro-industrial model has generated a unique model of food. Indeed, global food trends would have erased spatial differences in food consumption, a crucial issue for the geography of food. In a first section, the Ecuadorian diet is studied paying attention to its temporal evolution and to the similarities and differences compared to its neighbors (Colombia, Peru), South America and the world. In the second section, to understand the different diets within the Ecuadorian territory, the presence or absence of food in the cupboards of Ecuadorian households is evaluated with data from the ENIGHUR. Finally, in the third section, the interaction of the spatial behavior of the 27 products with the greatest spatial variation and importance, allows to generate five food regions characterized by a similar consumption. The study allows us to conclude that although the agro-industrial food model is part of the trends that have changed the consumption habits of Ecuadorians, there is a spatial expression of food regimes as regions. These regions respond to biophysical factors, but above all to social processes of the construction of the national space, which also have an imprint on the cultural elements.

Keywords: agro-food model, geography of food, urban rural linkages, Ecuador, food demand, food supply. 


\section{¿HACIA UN MODELO AGROALIMENTARIO ÚNICO? DIVERSIDAD E IDENTIDADES ESPACIALES EN EL CONSUMO DE ALIMENTOS EN ECUADOR}

\section{INTRODUCCIÓN}

La alimentación es uno de los componentes fundamentales de la cultura donde se expresan los conocimientos, preferencias y hábitos en el consumo de productos considerados alimenticios. Las relaciones entre estos productos y los consumidores evocan vínculos socioespaciales materiales construidos sobre significados (Alonso y Arjona, 2005). En las últimas décadas, el desarrollo de un modelo agroalimentario denominado "agroindustrial" ha llamado la atención en el contexto de la globalización (Malassis, 2006). Alrededor del mundo se observa tendencias alimentarias que privilegian el consumo de productos industrializados, ricos en grasas, azúcares y sales, lo que habría disminuido la importancia de productos considerados "tradicionales". Esta tendencia se desarrollaría, sobre todo, en las áreas urbanas las que de acuerdo con Moya (1989) tendrían, en el caso ecuatoriano, un cierto comportamiento autónomo frente a los campos. Esta idea contrasta con los fundamentos de la geografía de la alimentación que se interesa por la producción e intercambio de los productos alimentarios y por las prácticas alimentarias expresadas en la diferenciación espacial de los regímenes alimentarios (Charvet y Carré, 2008).

En este contexto es importante interesarse por el rol del espacio en la diferenciación de la alimentación. El presente estudio parte de la pregunta: ¿En Ecuador se ha construido un modelo único de alimentación borrando las diferencias regionales? Esta pregunta permite analizar la configuración espacial de la distribución de productos alimentarios y su vinculación con los espacios. Este tema es de particular interés al menos desde dos perspectivas. En primer lugar, comprender la demanda alimentaria es clave desde el enfoque de la producción agropecuaria ya que brinda luces sobre el funcionamiento espacial de este mercado. En segundo lugar, desde una perspectiva más amplia, la pregunta de investigación permite aproximarse a las demandas de alimentos desde un enfoque de seguridad alimentaria que no solo considera la existencia de efectivos, sino también la adecuación cultural entre las preferencias de la demanda social y la oferta alimentaria.

En un primer momento se aborda el patrón alimentario nacional lo que permite contextualizar la temática en una escala regional y global. En una segunda parte, se estudian los productos alimentarios presentes en los hogares ecuatorianos. Una tercera sección se enfoca en las diferencias regionales en el consumo alimentario para, en una cuarta sección, proponer una regionalización de los patrones alimentarios en Ecuador.

\section{DESARROLLO DEL TEMA}

Régimen alimentario ecuatoriano: entre tendencias globales y particularidades nacionales

La alimentación de los ecuatorianos ha llamado la atención de diversos investigadores, aunque se trata de un tema que históricamente ha estado en segundo plano en el debate público. Las operaciones estadísticas que permiten conocer los patrones alimentarios han sido espaciadas en el tiempo y limitadas en su cobertura demográfica. No es sino hasta la ENSANUT (Encuesta nacional de salud y nutrición) en el año 2012 (y que actualmente está en proceso de actualización) que se cuenta con datos con una mayor cobertura para el conjunto de la población, aunque se excluyen las personas de más de 60 años (INEC, 2013). La falta de otra operación estadística similar a la ENSANUT 2012 es un limitante en su uso, ya que no permite generar comparaciones temporales que muestren la evolución de las temáticas estudiadas. 


\section{¿HACIA UN MODELO AGROALIMENTARIO ÚNICO? DIVERSIDAD E IDENTIDADES ESPACIALES EN EL CONSUMO DE ALIMENTOS EN ECUADOR}

A nivel global, se han generado esfuerzos en la generación estadística sobre el sector agroalimentario, entre los que se destaca la recopilación de información secundaria realizada por la FAO por medio de su portal FAOSTAT. Se trata de una base de datos a largo plazo que permite estudiar la evolución del sector agroalimentario en los diversos países. En la temática del presente estudio las "Hojas de balances alimentarios" (Food balance sheets, FBS) son de particular importancia, ya que muestran la producción, importación, exportación, usos y consumo de 94 grupos de productos agroalimentarios organizados en 22 categorías.

De acuerdo con estos datos, el suministro de alimentos expresado en kilocalorías per cápita diarios en Ecuador ha seguido una evolución variable, aunque con una tendencia creciente en el largo plazo, la que es más fuerte en el período 1960-1980. Durante la "década perdida" (1980) el consumo de kilocalorías per cápita disminuyó, mientras que en los 1990 se recupera, para caer nuevamente en la primera década del nuevo milenio que presenta promedios de consumo similares a los de los años 1980. Para el año 2010 la tendencia ya marcaba un incremento del consumo y en el año 2011 marca el valor más elevado del período analizado (1961-2013). El consumo de kilocalorías per cápita en los primeros años de la segunda década del nuevo milenio son $13 \%$ más elevados que en los años 1960. No se trata solo de un cambio cuantitativo, sino también en la composición de los productos alimentarios consumidos.

Más allá del significado alimenticio expresado en kilocalorías, desde la perspectiva de los cambios alimentarios, el interés se dirige hacia la cantidad de productos consumidos expresada en el indicador Food supply quantity (cantidad de abastecimiento alimentario) que se expresa en kilogramos per cápita por año. En el caso ecuatoriano este indicador, al contrario que en las kilocalorías consumidas, muestra una disminución en su consumo. Esta paradoja se explica por la disminución de consumo de productos bajos en calorías como las frutas y el incremento de productos altos en calorías, como las carnes. En efecto, el régimen alimentario ecuatoriano se ha modificado en las últimas décadas, como se observa en el gráfico 1.

Se trata de una evolución que subraya lo dinámico y cambiante de los patrones de consumo de productos agroalimentarios. La mayoría de las categorías de productos han cambiado su orden de importancia y los que han permanecido muestran dinámicas en su consumo. Llama la atención la disminución del consumo de frutas, cuyo aporte porcentual pasa de casi el $40 \%$ en la década de 1960 a $25 \%$ en los primeros años de la década de 2010. Se trata de una disminución constante en cada decenio, una dinámica excepcional considerando que las otras categorías de productos presentan evoluciones que intercalan períodos de crecimiento y de decrecimiento. Este es el caso de los tubérculos que presentan un crecimiento en los años 1970 pero que disminuyen en el resto del período. Su aporte porcentual pasa de $12 \%$ a $4 \%$ entre las décadas de 1960 y 2010 . Las legumbres siguen una dinámica similar. En este caso, la década de crecimiento son los 1990, no obstante, su aporte porcentual es menor y pasa de $1.7 \%$ a $0.3 \%$ en el mismo período. Los vegetales pasan del $9 \%$ al $4.4 \%$ en el mismo período, con una disminución muy fuerte en los años 1970.

Al contrario, las categorías de productos que han aumentado su aporte porcentual, en orden de importancia, son: lácteos, cereales, carnes, bebidas alcohólicas, aceites vegetales y pescados y frutos de mar. Se trata de una evolución en consonancia con los cambios globales alimentarios (Kearney, 2010). En los países del Sur global se ha documentado un incremento en el consumo de lácteos en el marco de la globalización del consumo de leche (Wiley, 2007), así como de cárnicos y grasas (FAO, 2014). Llama la atención una ligera disminución en los datos de consumo de azúcar y endulzantes. Esta categoría de productos habría tenido su pico de consumo en los años 1980 desde cuando ha venido disminuyendo. El consumo alimentario en Ecuador marca así una evolución similar a los cambios globales, aunque con ciertas particularidades que pueden observarse en una comparación con sus vecinos, como lo muestra el gráfico 2. 


\section{¿HACIA UN MODELO AGROALIMENTARIO ÚNICO? DIVERSIDAD E IDENTIDADES ESPACIALES} EN EL CONSUMO DE ALIMENTOS EN ECUADOR

Gráfico 1. Consumo de productos agroalimentarios

en Ecuador en el período 1961-2013

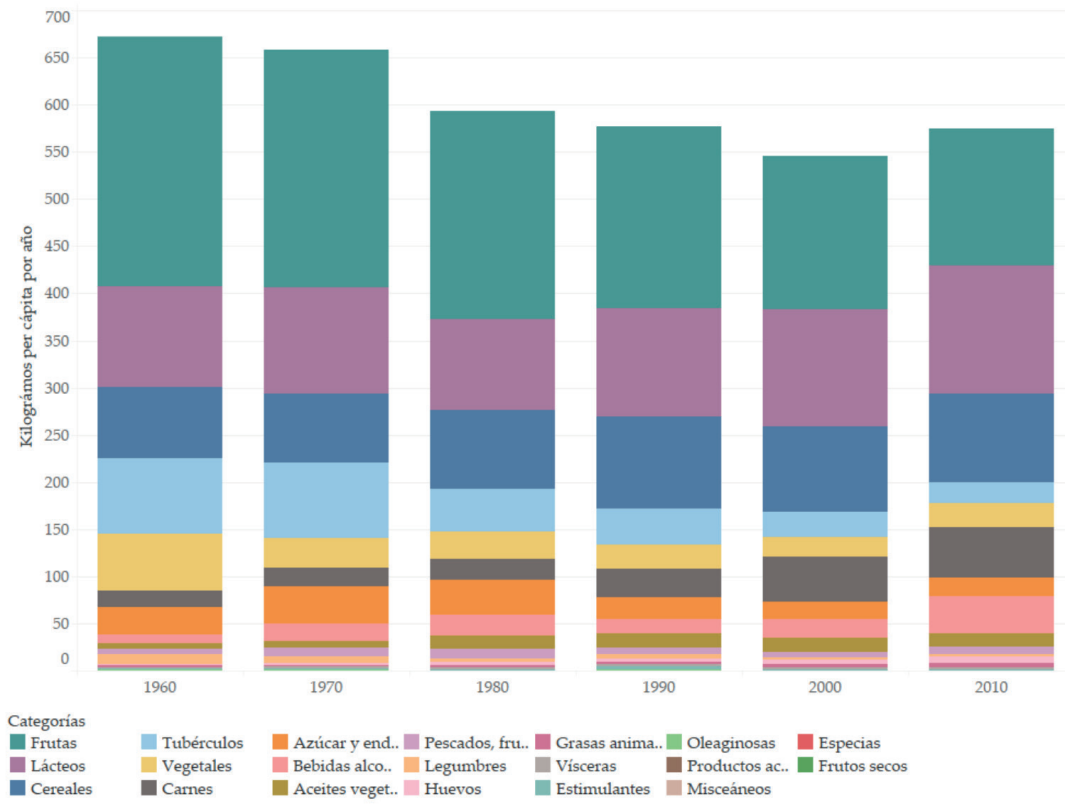

Fuente: Faostat, 2019.

Elaboración: Fernando Barragán Ochoa.

Gráfico 2. Consumo de productos agroalimentarios

en el período 2010-2013 por países

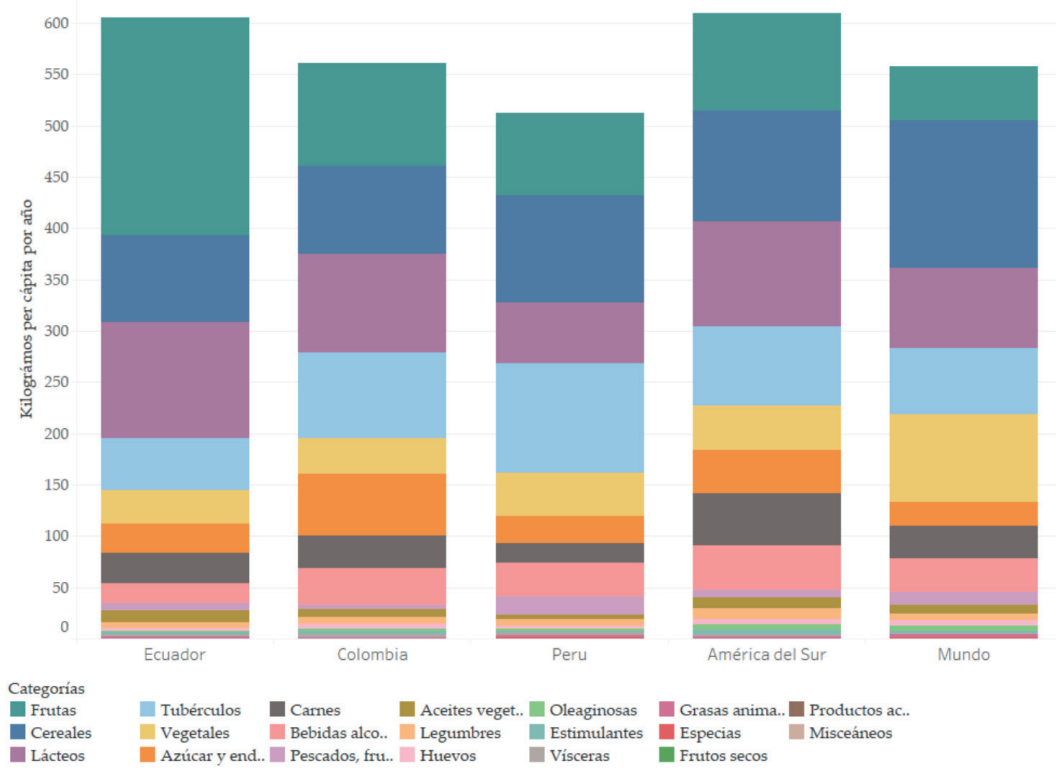

Fuente: Faostat, 2019

Elaboración: Fernando Barragán Ochoa. 


\section{¿HACIA UN MODELO AGROALIMENTARIO ÚNICO? DIVERSIDAD E IDENTIDADES ESPACIALES EN EL CONSUMO DE ALIMENTOS EN ECUADOR}

Una primera observación cuantitativa muestra que el consumo de productos agroalimentarios per cápita es más elevado en Ecuador que en sus países vecinos. En cuanto a la composición también se observan diferencias. A pesar de la disminución en el consumo de frutas en Ecuador (ver supra), este consumo es 2.1 veces mayor que el de Colombia, 2.6 que el de Perú y 4 que el consumo mundial. Este mismo caso se expresa en los lácteos, aunque en menor medida. Los ecuatorianos consumen $17 \%$ más que los colombianos, casi $200 \%$ más que los peruanos, $10 \%$ más que los pobladores de América del Sur y $44 \%$ más que el promedio mundial. Una situación similar es la de los aceites vegetales, mientras que en el resto de las categorías de productos agroalimentarios las situaciones presentan más matices. En las carnes, el consumo está por sobre el peruano y por debajo del colombiano, aunque los tres países están por debajo del consumo de América del Sur y del consumo mundial. En la categoría de azúcar y endulzantes el consumo en Ecuador es ligeramente superior que el de Perú, pero está por debajo del colombiano. En los pescados y frutos de mar el consumo ecuatoriano está por sobre el colombiano, pero muy por debajo del peruano. En los vegetales, tubérculos y cereales el consumo ecuatoriano es menor que el de sus vecinos.

Esta escala de análisis permite comprender las grandes evoluciones de los patrones alimentarios, sus especificidades y aspectos comunes. La lectura de los datos muestra que, si bien hay una tendencia común del comportamiento alimentario, los países continúan mostrando particularidades. Por citar algunos ejemplos, el alto consumo de pescado y frutos del mar en Perú sin duda responde a la localización de las mayores ciudades en el litoral, mientras que su alto consumo de tubérculos está vinculada la importancia histórica de este producto especialmente en las zonas andinas. El consumo elevado de leche en Ecuador se vincula con las políticas de apoyo a este producto (como la prohibición de importaciones), con el rol logrado por los pequeños productores y con la articulación entre producción en los campos y consumo urbano. Así también, las políticas de apoyo a la producción tienen un rol fundamental en el alto consumo de azúcares en Colombia.

Para profundizar el estudio del comportamiento alimentario desde un enfoque espacial es importante cambiar de escala. La mega-diversidad agraria y de paisajes geográficos al interior del Ecuador (Cepeda, Gondard, y Gasselin, 2007) lleva a pensar que además de una diferenciación en la escala nacional, habría también un componente regional en el consumo alimentario. La diversidad de platos típicos por regiones e incluso localidades fortalece esta hipótesis. La segunda parte aborda el consumo de productos alimentarios en Ecuador desde un enfoque espacial.

\section{Los productos alimentarios de los hogares ecuatorianos}

Este cambio de escala requiere también de un cambio en las herramientas metodológicas. Las estadísticas de FAOSTAT, de gran utilidad para las comparaciones internacionales, generan pocos aportes en una escala nacional. Es necesario recurrir a otras bases estadísticas. En este estudio utilizaremos la base de datos de la ENIGHUR ${ }^{3}$ que es una de las operaciones estadísticas más completas realizadas por el Instituto Nacional de Estadísticas y Censos (INEC) para comprender los presupuestos de los hogares. De entre los varios temas estudiados, se consideran los gastos de los hogares en productos alimentarios, lo que es analizado a través del módulo "Despensa" que se presenta como un inventario de

3 Está disponible para su descarga en el siguiente enlace http://anda.inec.gob.ec/anda/index.php/catalog/291 


\section{¿HACIA UN MODELO AGROALIMENTARIO ÚNICO? DIVERSIDAD E IDENTIDADES ESPACIALES EN EL CONSUMO DE ALIMENTOS EN ECUADOR}

alimentos y bebidas en las despensas o alacenas de los hogares. En el presente estudio quedan excluidos entonces los alimentos consumidos fuera del hogar.

Si bien el procesamiento de datos de la ENIGHUR permite determinar la cantidad de los productos consumidos, en esta investigación el interés se dirige al estudio de la presencia o ausencia de productos alimentarios en las alacenas de los hogares ecuatorianos. Desde el punto de vista metodológico es importante recalcar que esta operación estadística no busca estudiar el significado alimenticio de los productos, sino más bien la composición de los gastos de los hogares. Los datos de la ENIGHUR no son comparables con los de los balances alimentarios de la FAOSTAT, analizados previamente. Para ejemplificar, la ENIGHUR muestra que un hogar consumió una cantidad determinada de queso, yogur y leche en líquido, pero no estudia el aporte de cada uno de estos productos a la cifra total de consumo de lácteos, como lo hace los datos de la FAOSTAT. Por otra parte, el nivel de desglose en la ENIGHUR es mucho mayor que el de FAOSTAT. De hecho, se considera más de 700 productos que forman parte de las alacenas de los hogares ecuatorianos. Por ejemplo, se diferencia once tipos de quesos.

Se enlistan a continuación los cincuenta productos con mayores porcentajes de presencia en las alacenas de los hogares ecuatorianos.

Tabla 1

Productos alimentarios con mayor presencia en las alacenas de los hogares ecuatorianos

\begin{tabular}{|c|c|c|c|}
\hline 1. Sal refinada & 14. Atún en aceite vegetal & 27. Arroz flor & 40. Col de seda o col verde \\
\hline 2. Azúcar refinada & 15. Pimiento & 28. Naranja & 41. Canguil \\
\hline 3. Aceite de palma & 16. Zanahoria amarilla & 29. Limón sutil & $\begin{array}{l}\text { 42. Leche cruda (no } \\
\text { pasteurizada) }\end{array}$ \\
\hline 4. Fideos & 17. Fideos tallarín & 30. Fréjol tierno & 43. Plátano maduro \\
\hline 5. Arroz blanco & 18. Pan corriente trigo & 31. Huevos criollos & 44. Salsa de tomate \\
\hline 6. Cebolla paiteña & 19. Plátano verde & 32. Aceite achiote & 45. Harina de maíz \\
\hline $\begin{array}{l}\text { 7. Café soluble } \\
\text { e instantáneo }\end{array}$ & 20. Fréjol seco (porotos) & 33. Arveja tierna & 46. Brócoli \\
\hline 8. Papa chola & 21. Margarina & $\begin{array}{l}\text { 34. Pechuga, alas y piernas } \\
\text { de pollo }\end{array}$ & 47. Pechuga de pollo \\
\hline 9. Tomate riñón & 22. Aliños preparados & 35. Ajo en pepa (pelado) & 48. Harina de cebada (máchica) \\
\hline 10. Avena & 23. Manzana & 36. Tomate de árbol & 49. Habas tiernas \\
\hline 11. Huevos de granja & 24. Leche entera funda & 37. Queso de mesa & 50. Sardinas en salsa de tomate \\
\hline 12. Lenteja & 25. Banano (guineo) & 38. Cocoa para bebidas & \\
\hline 13. Harina de trigo & 26. Cebolla blanca & 39. Yuca & \\
\hline
\end{tabular}

Fuente: ENIGHUR, 2012.

Elaboración: Fernando Barragán Ochoa.

La presencia de estos productos difiere en los hogares según diversas variables. Se ha documentado, por ejemplo, como el consumo de leche y quesos varía en función de los quintiles económicos (Barragán Ochoa, 2010). El interés en el presente estudio se dirige al componente espacial de esta variación, dejando de lado los otros factores para su comprensión. 


\section{¿HACIA UN MODELO AGROALIMENTARIO ÚNICO? DIVERSIDAD E IDENTIDADES ESPACIALES EN EL CONSUMO DE ALIMENTOS EN ECUADOR}

\section{Un consumo diferenciado en el espacio}

El componente espacial del consumo alimentario es una variable fundamental desde la perspectiva de la seguridad y soberanía alimentaria. Las relaciones entre espacios y productos alimentarios se construyen a lo largo del tiempo y es parte de la cultura que se expresa en diversas escalas. Así también, es un elemento esencial en la comprensión de los circuitos de aprovisionamiento, encuentra su aplicación a través de políticas públicas que mejoren la eficiencia y la justicia de estas relaciones que atraviesan espacios urbanos y rurales y generan la interacción de una diversidad de actores.

El comportamiento del consumo de productos alimentarios puede ser diverso. Desde productos en los que el espacio no sea una variable importante (como la sal o el azúcar, que tienen una amplia distribución por todo el territorio), hasta productos profundamente arraigados en regiones o localidades, los que en los últimos años han sido revalorizados a través de modelos agroalimentarios que vinculan la calidad con el origen geográfico y el saber-hacer de los productores locales, como los sistemas agroalimentarios localizados (SYAL) (Muchnik, Marie, y Leclerc, 2010).

Se presentan a continuación varios ejemplos de modelos estadístico-espaciales de la presencia de los productos agroalimentarios en las alacenas de los hogares ecuatorianos. Estos ejemplos han sido seleccionados en consideración de dos criterios: su importancia en porcentaje y su variabilidad interprovincial.

En el mapa 1 se han considerado seis productos agroalimentarios que ejemplifican diversas modalidades de la expresión espacial de su presencia en las alacenas de los hogares. Para asegurar su comparabilidad todos los mapas están construidos sobre la base de una misma escala gráfica constituida por ocho intervalos de una amplitud de $7.5 \%$ en un rango comprendido entre $20 \%$ y $80 \%$. Este tipo de cartografía, representado por algoritmos de cartografía estadística, ${ }^{4}$ puede ser generada para cada uno de los 700 productos considerados en la ENIGHUR. Los seis seleccionados permiten realizar algunas lecturas espaciales.

- El arroz blanco está ampliamente distribuido por todas las provincias, aunque es en el sur del país donde su presencia en los hogares está más expandida. En las provincias de la sierra central (Bolívar, Tungurahua, Chimborazo y Cotopaxi) y en Napo y Pastaza de la Amazonía la presencia de este producto tiene los menores porcentajes a nivel nacional. Sin embargo, esto no necesariamente significa una presencia menor de arroz en estas provincias. Debido a la subdivisión del arroz en varios tipos (blanco, flor, viejo...) se introduce algunos sesgos en el análisis. Por ejemplo, Napo donde solo el $31 \%$ de los hogares tienen arroz blanco en su alacena, tiene el porcentaje más elevado en la presencia de arroz flor (36 \%). De hecho, considerando la variedad de tipos de arroces la variabilidad interprovincial es menor. Se trata de un producto de consumo generalizado en el territorio ecuatoriano.

- El consumo de la avena muestra una concentración en las provincias de la sierra central que se extiende hacia el sur (Cañar, Azuay). A diferencia del arroz blanco su distribución en el país presenta rupturas. El paso entre Sierra y Costa constituye una ruptura en el consumo de este producto que está presenta en el $55 \%$ de los hogares de la Sierra y en solo el $27 \%$ de los hogares de la Costa. Las rupturas entre Sierra y Amazonía son menos perceptibles que las mencionadas previamente. 
Mapa 1. Ejemplos de expresión espacial de la varianza en el consumo de productos agroalimentarios
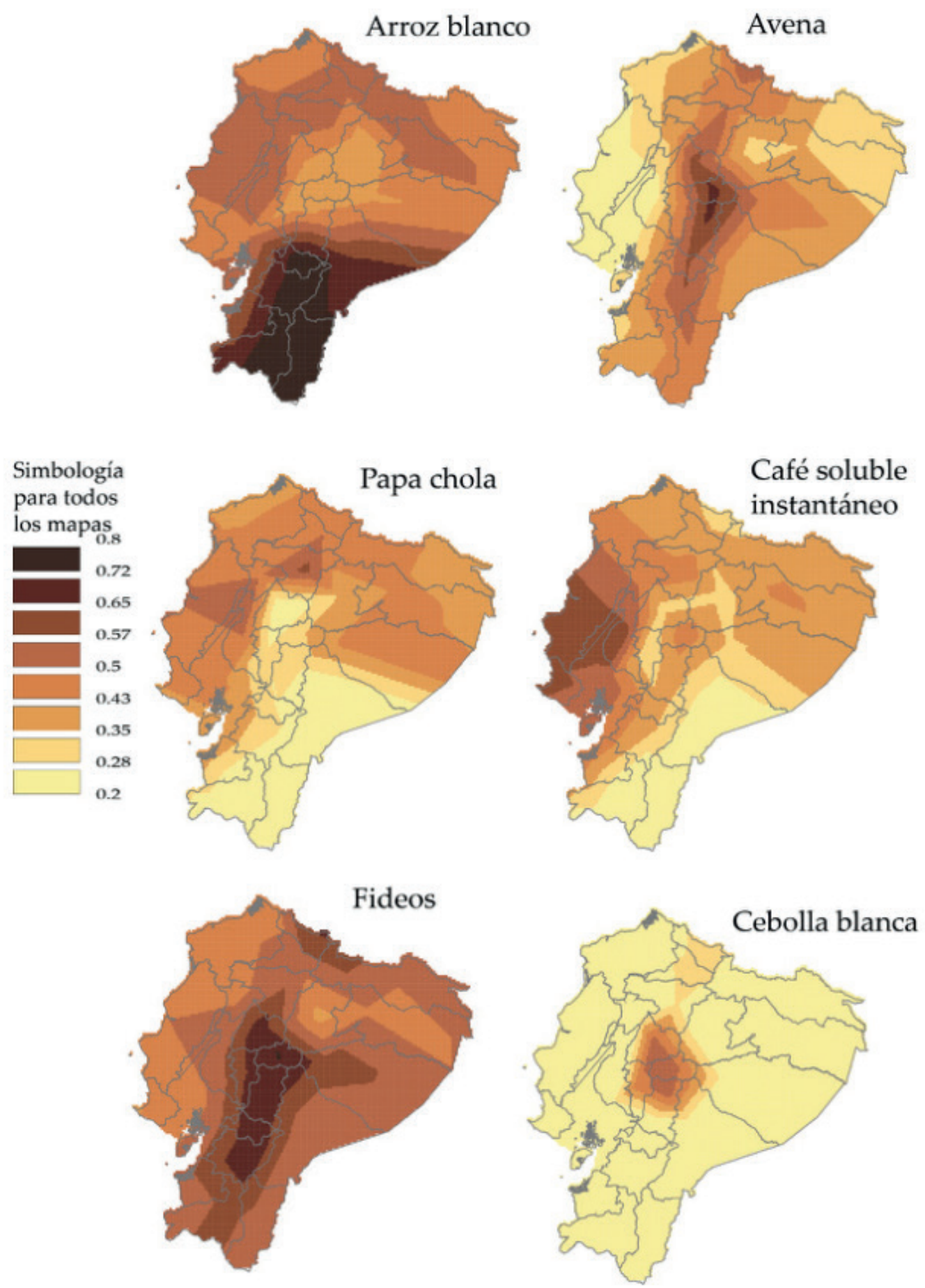

Fuente: ENIGHUR, 2012.

Elaboración: Fernando Barragán Ochoa. 


\section{¿HACIA UN MODELO AGROALIMENTARIO ÚNICO? DIVERSIDAD E IDENTIDADES ESPACIALES} EN EL CONSUMO DE ALIMENTOS EN ECUADOR

- La presencia de papa chola en las despensas de los hogares se expresa en una forma particular. La distribución de las provincias de mayor consumo forma una media luna que inicia en Napo, se extiende por el norte atravesando los Andes para nuevamente bajar a las Ilanuras de la Costa y llegar hasta el Guayas. En este recorrido sus puntos más elevados están en Pichincha y Manabí. Este comportamiento particular que une provincias de la Sierra, Costa y Amazonía se explica también en parte por un sesgo metodológico similar al del arroz blanco. La ENIGHUR considera la diversidad de papas como categorías diferentes.

- El café soluble instantáneo tiene también una amplia distribución en el territorio ecuatoriano. Sin embargo, destacan ciertas provincias en las que, por condiciones locales y regionales, se consume otro tipo de cafés o de bebidas y la presencia del café soluble en los hogares de estas provincias disminuye. Este es el caso del eje formado por Loja, Zamora Chinchipe y Morona Santiago en el sur y sureste del país, donde el consumo del café tostado molido tiene una gran importancia. Bolívar presenta también un comportamiento local que lo diferencia de sus vecinos en la presencia del café soluble, donde este producto está en el $28 \%$ de los hogares frente al $46 \%$ de sus vecinos inmediatos.

- Los fideos, en el puesto cuarto de los alimentos de mayor presencia en las alacenas (ver tabla 1), tiene una presencia en todas las provincias. Únicamente en Napo su presencia se registra en menos del $40 \%$ de los hogares, mientras que en la Sierra Centro y Sur está por sobre el $70 \%$.

- La cebolla blanca muestra un comportamiento espacial local. En las provincias de la sierra centro su presencia en las alacenas de los hogares supera el $50 \%$. Este alto consumo se extiende, aunque en menor medida, hacia las provincias de la sierra norte donde está en aproximadamente uno de cada tres hogares. Su expansión hacia las tierras bajas tanto de la Amazonía como de la Costa es menor. Hacia el sur de la sierra centro se presenta una discontinuidad en el límite entre Chimborazo y Cañar. En las provincias de Cañar, Azuay y Loja la cebolla blanca está presente en únicamente el $3 \%$ de los hogares.

La lectura espacial de la presencia de estos seis productos agroalimentarios muestra diversas situaciones. Desde productos de amplia distribución en el territorio, hasta otros que están concentrados en pocas provincias. Se han observado también las diferencias entre regiones (en la avena, por ejemplo), pero también productos que muestran otras geografías. Más allá de los casos particulares, el objetivo en el presente estudio es determinar cómo la distribución de la presencia de alimentos en los hogares permite dibujar regiones en las que se expresen patrones alimentarios similares. La siguiente parte aborda este desafío.

\section{Unos patrones alimentarios regionales}

Se ha observado en este estudio las particularidades y elementos comunes del consumo agroalimentario ecuatoriana con respecto a sus vecinos y en contexto más amplios de América del Sur y el mundo (gráfico 2). Así también se han listado los productos que con mayor frecuencia forman parte de las alacenas de los hogares ecuatorianos (tabla 1) y se ha analizado la distribución espacial de seis ejemplos (mapa 1). Sin embargo, estos análisis todavía no son suficiente para proponer una regionalización de los patrones alimentarios. Para esto es necesario generar una interacción entre los datos sobre la distribución de los productos agroalimentarios. 


\section{¿HACIA UN MODELO AGROALIMENTARIO ÚNICO? DIVERSIDAD E IDENTIDADES ESPACIALES EN EL CONSUMO DE ALIMENTOS EN ECUADOR}

¿Qué productos considerar? Como se ha mencionado, la ENIGHUR permite el acceso a datos para más de 700 productos agroalimentarios presentes en las alacenas de los hogares ecuatorianos. Este número de variables generaría matrices poco manejables con datos que aportarían poco al objetivo planteado. Interesa centrar el análisis en los productos con una alta diferenciación espacial y que su presencia en los hogares sea importante. Los estadísticos de coeficiente de variación y media permiten determinar qué productos cumplen con los requisitos planteados, como se observa en el gráfico 3.

Gráfico 3. Estadísticos de distribución de los productos agroalimentarios en las provincias en Ecuador

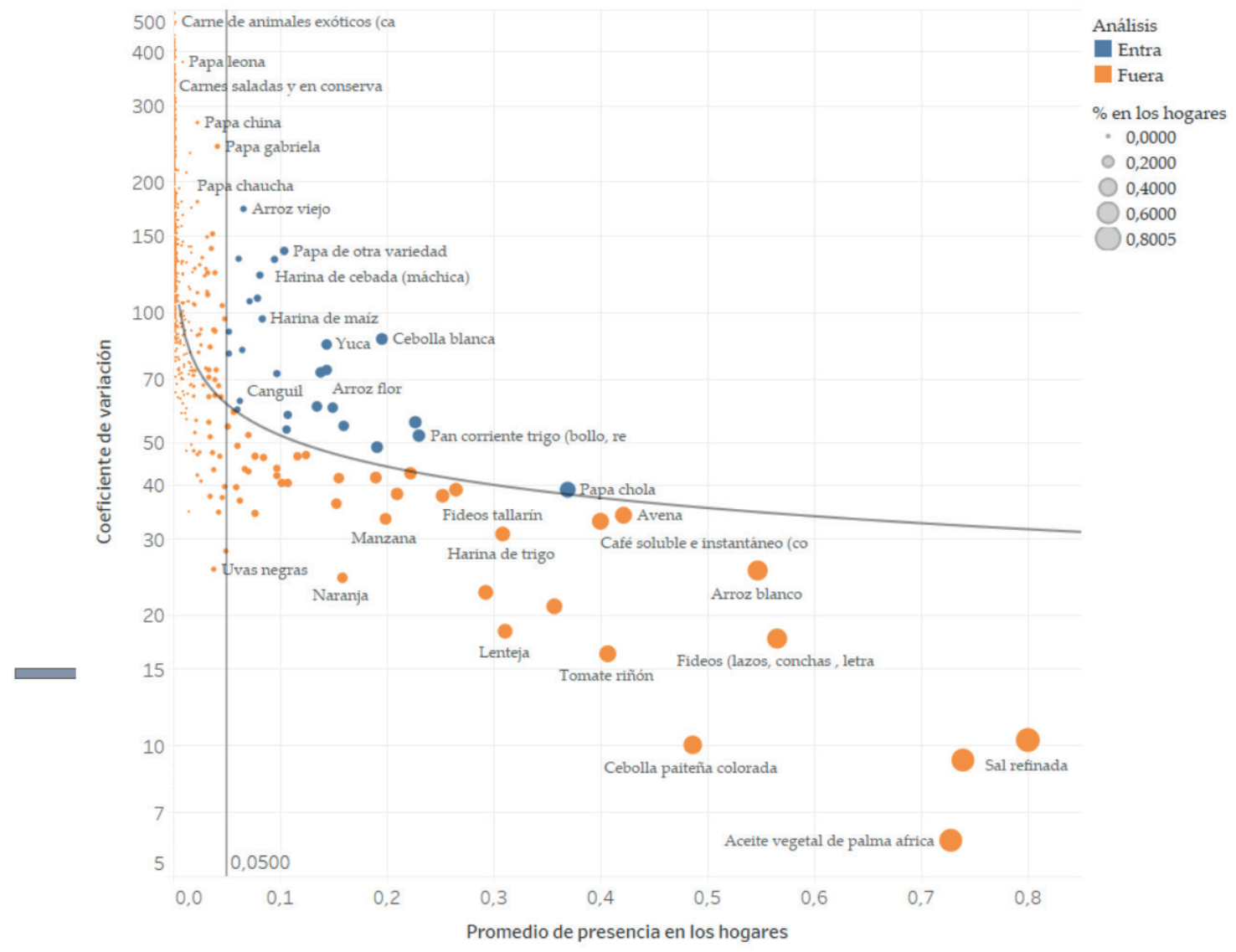

Fuente: ENIGHUR, 2012.

Elaboración: Fernando Barragán Ochoa.

En el gráfico 3, el eje de las coordenadas (y) muestra, en una escala logarítmica para facilitar la visualización de los datos, el coeficiente de variación de la distribución de los productos por provincias y en las abscisas (x), el promedio de la presencia de los productos en las alacenas de los hogares ecuatorianos. La curva de tendencia potencial se ajusta bastante bien a la distribución de los datos caracterizada por una multitud de productos con bajos valores de presencia en los hogares y altos de coeficiente de variación. 


\section{¿HACIA UN MODELO AGROALIMENTARIO ÚNICO? DIVERSIDAD E IDENTIDADES ESPACIALES EN EL CONSUMO DE ALIMENTOS EN ECUADOR}

De esta multitud de puntos (que representan a los productos agroalimentarios), interesan sobre todo los que están por sobre la curva de tendencia, lo que permite centrarse en los productos que mayor variación espacial tienen. Así también, interesan los productos cuya representatividad sea importante en los hogares, por lo que se ha descartado los productos cuya presencia en los hogares es menor al $5 \%$. Los productos incluidos en el análisis son señalados a través de un color azul en el gráfico 3.

Se obtiene así una base de datos que muestra el porcentaje de hogares por cada provincia en los que se encuentran cada uno de los 27 productos agroalimentarios señalados para el análisis. Una lectura del comportamiento espacial de esta matriz es posible a través de la aplicación del método de estadística espacial multivariable "Clasificación Ascendente Jerárquica" que permite la generación de perfiles que agrupan unidades espaciales (en este caso provincias) que tienen un comportamiento similar en la expresión de diferentes variables (los 27 productos analizados). El producto de este análisis se presenta en el mapa 2.

\section{Mapa 2. Perfiles alimentarios en Ecuador}

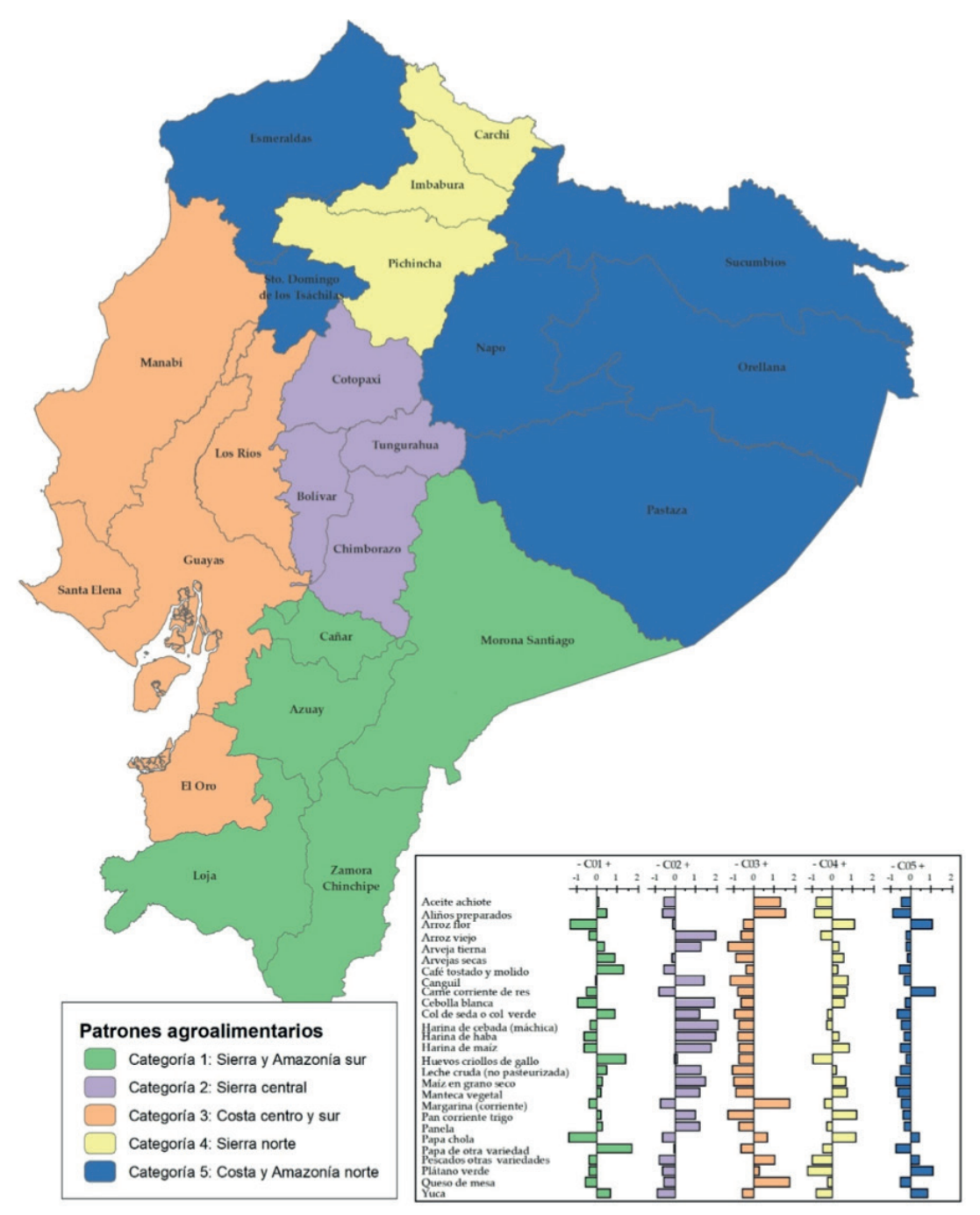

Fuente: ENIGHUR, 2012

Elaboración: Fernando Barragán Ochoa 


\section{¿HACIA UN MODELO AGROALIMENTARIO ÚNICO? DIVERSIDAD E IDENTIDADES ESPACIALES EN EL CONSUMO DE ALIMENTOS EN ECUADOR}

Como se observa en el mapa 2, se ha seleccionado la clasificación en cinco categorías, cuyo orden resulta del logaritmo de cálculo pero que no tiene ningún significado en su interpretación. Se trata de categorías cualitativas no ordinales. La primera observación es la coherencia espacial de las clases conformadas. Todas agrupan provincias contiguas y solo una (la categoría 5) presente una discontinuidad espacial. Se analizan a continuación las principales características de estas categorías.

\section{Categoría 1: Sierra y Amazonía sur}

Agrupa las provincias de Cañar, Azuay, Loja, Morona Santiago y Zamora Chinchipe. Es una de las dos categorías, junto con la 5, que sobrepasa el alcance de las regiones consideradas naturales y establece un vínculo entre Sierra y Amazonía en el sur del país. Los productos que más destacan en esta agrupación son el café tostado molido, los huevos criollos y la presencia de papas de variedades locales por sobre otras variedades como la papa chola cuya presencia en los hogares es menor en comparación con el resto del país. Así también las arvejas secas, la leche cruda y la yuca forman parte de los productos que caracterizan a esta categoría.

Categoría 2: Sierra central

Esta categoría se encuentra en la Sierra Central estableciendo un patrón alimentario común entre las provincias de Cotopaxi, Tungurahua, Bolívar y Chimborazo. En esta unidad destacan los productos andinos como la arveja tierna, la cebolla blanca, la col, el haba y maíz en forma de harina, la leche, el pan, entre otros. El arroz también tiene una presencia importante en los hogares de esta provincia, pero en su variedad "viejo" que proviene de un proceso de secado. Este tipo de arroz se cocina mejor en las alturas de los Andes. Los productos que están subrepresentados en estas provincias son el achiote, los aliños, la margarina, la carne de res, la yuca, el plátano verde y los pescados.

Categoría 3: Costa centro y sur

A esta categoría pertenecen todas las provincias de la Costa con excepción de Santo Domingo de los Tsáchilas y Esmeraldas. Conforma una gran unidad espacial continua que agrupa un importante porcentaje de la población ecuatoriana. Sus características son, en gran medida, casi opuestas a las del perfil anterior (categoría 2). Se plantea así una diferenciación entre los patrones alimentarios de la sierra central y la Costa. Los productos con una mayor representación son el achiote, los aliños, la margarina, la papa chola, los pescados, el queso de mesa y el plátano verde. Por el contrario, los productos agroalimentarios subrrepresentados son en general los que se producen en la Sierra, así: las arvejas, la cebolla blanca, la col, cebada, haba, huevos criollos, leche "cruda", pan, yuca, entre otros.

Categoría 4: Sierra norte

En la categoría 4 están las provincias de la Sierra norte, desde Pichincha, pasando por Imbabura, hasta el Carchi. La presencia de productos agroalimentarios en estas provincias es cercana al promedio nacional, aunque se observan algunas particularidades que merecen ser mencionadas. El arroz con mayor presencia en estas provincias es la variedad "flor". Así también la presencia de pan y papa chola son características que diferencian esta categoría del resto del territorio nacional. El plátano verde, los pescados, los huevos criollos, los aliños preparados, el achiote y el arroz viejo están poco presentes en las alacenas de los hogares de estas provincias.

Categoría 5: Costa y Amazonía norte

Esta categoría llama la atención desde el punto espacial ya que a diferencia de las previas se presenta en dos áreas separadas de dos de las regiones consideradas naturales. En la Costa está presente en las provincias de Santo Domingo de los Tsáchilas y Esmeral- 


\section{¿HACIA UN MODELO AGROALIMENTARIO ÚNICO? DIVERSIDAD E IDENTIDADES ESPACIALES}

EN EL CONSUMO DE ALIMENTOS EN ECUADOR

das, mientras que en la Amazonía está en las provincias de Sucumbíos, Napo, Orellana y Pastaza. Se trata de conjuntos espaciales similares desde el punto de visto biofísico y que históricamente han seguido procesos socioespaciales similares caracterizados por la política de colonización. Se trata de factores a considerar al estudiar la presencia de patrones alimentarios similares en áreas discontinuas. Esta categoría se caracteriza por la presencia de arroz flor, carne de res, plátano verde, yuca y en menor medida pescados de diversas variedades y papa chola. Por otra parte, productos agroalimentarios como los aliños, papas de otras variedades y los quesos de mesa están subrepresentados, como también una diversidad de productos de la sierra central.

\section{CONCLUSIONES}

\section{La importancia del espacio en la comprensión de los regímenes alimentarios}

Este estudio ha partido de la interrogante sobre la pertinencia de considerar los espacios para la comprensión de la distribución de los patrones alimentarios. La difusión del modelo agroindustrial en los sistemas agroalimentarios a nivel global, desde ciertas perspectivas teóricas, significaría que las diferencias regionales en los patrones alimentarios pasarían a un segundo plano. Sin embargo, el análisis presentado muestra una situación diferente. Si bien la alimentación en Ecuador forma parte de patrones de cambio globales entre los que se destaca una mayor importancia a los productos agroindustriales que incrementan el consumo de azúcares, sales y grasas, este modelo no ha logrado borrar diferencias existentes que deben entenderse en sus relaciones con los espacios locales y regionales.

En efecto, el patrón alimentario ecuatoriano se diferencia del de sus vecinos (Perú y Colombia) y aún más del de América del Sur y del mundo. Este nivel no es suficiente para comprender los patrones agroalimentarios en Ecuador. Las estadísticas de la ENIGHUR son una fuente de datos primordial al momento de estudiar los patrones alimentarios en escalas regionales. Los diversos productos agroalimentarios dibujan geografías diversas en su consumo en los hogares. Se podría analizar más de 700 mapas sobre la distribución de los productos agroalimentarios en los hogares ecuatorianos. Sin embargo, el mayor aporte es observar como la interacción de estos productos muestra un comportamiento regional de la alimentación. Se observa que los patrones alimentarios están arraigados en los espacios con una coherencia regional que llama la atención. No se trata sin embargo de las regiones consideradas naturales, aunque esta configuración ayude a comprender la distribución de los patrones alimentarios. Dos de las cinco categorías espaciales generadas en este estudio sobrepasan los límites de estas regiones "naturales" y evocan procesos históricos socioespaciales que están en el origen de la construcción de estos espacios. Así, a través de la alimentación se entiende la lógica regional de la construcción del espacio y se demuestra que la geografía tiene mucho que aportar en el estudio de estructuras y dinámicas agroalimentarias.

Más allá de las certezas, el presente estudio abre diversas perspectivas para profundizar la investigación. Desde esta perspectiva es interesante estudiar la interacción entre la estructura regional resultante del estudio de los patrones alimentarios y las estructuras espaciales de la agro-producción. ¿Los patrones alimentarios de consumo guardan relación con la estructura agro-productiva? En algunos casos, como la sierra central, se observa que varios de los productos que tienen importancia desde el lado del consumo, lo tienen también desde la producción en los campos de estas provincias. Al mismo tiempo, se observa que 


\section{¿HACIA UN MODELO AGROALIMENTARIO ÚNICO? DIVERSIDAD E IDENTIDADES ESPACIALES EN EL CONSUMO DE ALIMENTOS EN ECUADOR}

productos de otras regiones, pero con variantes específicas, como el arroz viejo, forman parte importante del consumo. Una amplia perspectiva de investigación debe vincularse con las modalidades de aprovisionamiento alimentario desde una perspectiva de seguridad alimentaria, que, además de la existencia de los alimentos, se interese por su pertinencia cultural, tema al que la presente investigación aporta.

Desde el enfoque se salud pública, el consumo de alimentos puede relacionarse con la distribución de diversos tipos de enfermedades como las cardiovasculares. Un análisis que relacione los patrones alimentarios con las patologías, sin duda sería un gran aporte que permita generar políticas públicas que fomenten la salud preventiva.

Finalmente, una tercera perspectiva de investigación se relaciona a los vínculos entre productores rurales y consumidores urbanos. ¿De qué manera los patrones alimentarios identificados pueden ser una herramienta para fomentar una interacción urbano-rural que favorezca a los productores de los campos?

Estas perspectivas quedan abiertas para futuras investigaciones, pero queda claro que las variables espaciales son aspectos esenciales, ya que, como se ha demostrado, la configuración de los patrones alimentarios está basada en una interacción entre productos agroalimentarios y espacios.

\section{BIBLIOGRAFÍA}

Adoum, A. (ed.). (s/f). Seguridad alimentaria y nutricional en el Ecuador. Construyendo la soberanía alimentaria. Quito, Ecuador: Ministerio Coordinador de Desarrollo Social / AECID / FAO.

Alonso, L. E., y Arjona, P. (2005). La era del consumo (1. ${ }^{a}$ ed.). Madrid: Siglo XXI.

Barragán Ochoa, F. (2015). Las redes de abastecimiento en el contexto de la seguridad alimentaria: el caso de la leche en Ecuador. En Seguridad alimentaria: responsabilidad de los gobiernos autónomos descentralizados provinciales. Quito, Ecuador: Abya-Yala.

Barragán Ochoa, F. (2017). Les petits producteurs, les villes et le lait: Défis du ravitaillement alimentaire dans les Andes du nord de l'Equateur. París, Francia: Université Paris 1 Panthéon Sorbonne.

Cepeda, D., Gondard, P., y Gasselin, P. (2007). Mega diversidad agraria en el Ecuador: disciplina, conceptos y herramientas metodológicas para el analisis-diagnóstico de micro-regiones, 29-54. Quito, Ecuador: Mosaico agrario / SIPAE / IRD / IFEA.

Charvet, J. P. (2009). L'alimentation dans le monde: Mieux nourrir la planète. París, Francia: Larousse.

Claval, P. (2012). Géographie culturelle: une nouvelle approche des sociétés et des milieux. París, Francia: A. Colin.

Da Silva, C. A. (2009). La configuración de los circuitos «de proximidad» en el sistema alimentario: tendencias evolutivas. Documents d'anàlisi geogràfica, (54), 011-32.

Delgado, C. L. (2003). Rising Consumption of Meat and Milk in Developing Countries Has Created a New Food Revolution. The Journal of Nutrition, 133(11), 3907S-3910S.

Delgado, C., Rosegrant, M., Steinfeld, H., Ehui, S., y Courbois, C. (eds.). (1999). Livestock to 2020: the next food revolution. Washington, DC, EE. UU.: FAO / ILRI / IFPRI.

Dubois, S. (2010). Le défi alimentaire: étude géopolitique et géoéconomique des agricultures mondiales. En Collection Major. París, Francia: Presses universitaires de France.

Enríquez Bermeo, F. (ed.). (2015). Seguridad alimentaria: responsabilidad de los gobiernos autónomos descentralizados provinciales (1. ${ }^{a}$ ed.). Quito, Ecuador: Abya-Yala.

FAO. (2011). Food, agriculture and cities. The challenges of food and nutrition security, agriculture and ecosystem management in an urbanizing world. Recuperado de http://www.fao.org/3/aau725e.pdf

FAO. (2014). El estado mundial de la agricultura y la alimentación. La innovación en la agricultura. 


\section{¿HACIA UN MODELO AGROALIMENTARIO ÚNICO? DIVERSIDAD E IDENTIDADES ESPACIALES EN EL CONSUMO DE ALIMENTOS EN ECUADOR}

García, A. G. R., Pérez, M. A. C., Miranda, C. A. R., y García, A. E. (2017). La soberanía alimentaria. El enfoque desde los territorios y las redes agroalimentarias. SAPIENTIAE, 2(2), 127-147.

Ghersi, G., Rastoin, J.-L., y Schutter, O. D. (2010). Le système alimentaire mondial : Concepts et méthodes, analyses et dynamiques. Versailles: Quae éditions.

INEC (2013). Encuesta nacional de salud y nutrición: ENSANUT-ECU 2011-2013 (1a. ed). Quito, Ecuador: INEC / Ministerio de Salud Pública.

Kearney, J. (2010). Food consumption trends and drivers. Philosophical Transactions of the Royal Society of London B: Biological Sciences, 365(1554), 2793-2807. https://doi.org/10.1098/ rstb.2010.0149

León Velasco, J. B., Moya, A., y Peltre, P. (eds.). (1989). Flujos geográficos en el Ecuador: intercambios de bienes, personas e información. Quito: Corporación Editora Nacional / Colegio de Geógrafos del Ecuador.

Llaguno, S., y Naynee, S. (2014). Estudio preliminar de la alimentación de la población ecuatoriana. Recuperado de http://helvia.uco.es/xmlui/handle/10396/11532

Moreno, M. (2013). Perfil Nutricional de Ecuador, junio, 2001. FAO. Roma. Apertura, 11(10).

Moya, L. (1989). Flujos de productos alimenticios y mercados en el Ecuador. 1.

Muchnik, J., Marie, C. de S., \& Leclerc, B. (2010). Le temps des Syal: Techniques, vivres et territoires. Recuperado de http://public.eblib.com/choice/publicfullrecord.aspx? $p=3399237$

Neira Mosquera, J. A. (2014). Estudio de la alimentación de la población inmigrante ecuatoriana en España. Recuperado de http://helvia.uco.es/xmlui/handle/10396/11533

Peltre-Wurtz, J. (2004). Luchar para comer: estrategias familiares para la alimentación en sectores populares. Quito, Ecuador: CEDIME / IRD / Abya-Yala.

Sack, R. D. (1992). Place, modernity, and the consumer's world: a relational framework for geographical analysis. Baltimore: Johns Hopkins University Press.

Schmidhuber, J., y Shetty, P. (2005). The nutrition transition to 2030. Why developing countries are likely to bear the major burden. Acta agriculturae scand section c, 2(3-4), 150-166.

Sorre, M. (1952). La géographie de l'alimentation. Annales de Géographie, 61(325), 184-199. https:// doi.org/10.3406/geo.1952.13393

Valencia-Valero, R. G., y Ortiz-Hernández, L. (2014). Disponibilidad de alimentos en los hogares mexicanos de acuerdo con el grado de inseguridad alimentaria. salud pública de méxico, 56(2), 154-164.

Wiley, A. S. (2007). The Globalization of Cow's Milk Production and Consumption: Biocultural Perspectives. Ecology of Food and Nutrition, 46(3-4), 281-312. https://doi.org/10.1080/036 70240701407657 